\title{
Rare Yeasts: Emerging Threat in Immunocompromised Patients
}

\author{
Mahir Kapmaz¹, Süda Tekin¹, Özlem Doğan¹, Şiran Keske², Füsun Can¹, Önder Ergönül1 \\ 1 Department of Infectious Diseases and Clinical Microbiology, Koç University School of Medicine, İstanbul, Turkey \\ 2 Department of Infectious Diseases and Clinical Microbiology, V.K.V. American Hospital, İstanbul, Turkey
}

\begin{abstract}
Fungemia due to the yeasts other than Candida is a clinically rare, but still emerging entity. The clinical terminologies of rare opportunistic yeast bloodstream infections (ROYBSIs) or micafungin breakthrough fungemia (MBF) or echinocandin non-susceptible yeast (ENSY) refer mostly to the same entity. Clinicians should be alert about the early distinction. ROYBSIs are mostly resistant to echinocandins and should be taken into consideration when yeast growth in blood culture of a patient previously treated with an antifungal, especially an echinocandin. Apart from molecular methods, MALDI-TOF might be considered as the most useful commercial system for identification of ROYBSIs. A combination of L-AmB and voriconazole is the appropriate empirical therapy for MBF, especially in neutropenic patients. Keywords: Candidemia, Non-Candida, yeast, fungemia, echinocandin resistant, breakthrough, non-susceptible, bloodstream
\end{abstract}

\section{INTRODUCTION}

I nfections of the yeasts other than Candida spp. are rare, but an emerging clinical entity. Clinicians should be alert for early diagnosis. Early distinction of non-Candida 1 yeasts is important because of varying antifungal susceptibilities, higher mortality of 50-80\% particularly among immunocompromised patients (1). These yeasts include Basidiomycetes (Cryptococcus, Trichosporon, Rhodotorula, and Malassezia species) and the non-Candida Ascomycetes (such as Hansenula, Saccharomyces, Saprochaete capitata, and Saprochaete clavata). Basidiomycetes are less susceptible to all antifungal drugs compared to Ascomycetes due to the difference in their cell wall integrity (2). Cryptococcus, Trichosporon, Rhodotorula, and Saccharomyces species are intrinsically resistant to echinocandins, and Trichosporon species could also be resistant to amphotericin (3). Moulds such as Fusarium spp., Scedosporium spp., or Exophiala spp. may cause to fungemia (4). These moulds and Cryptococci that was the leading second most common yeast after Candida spp., were not included in this review.

Several non-Candida yeasts were reported to be resistant to echinocandins, as a distinction to Candida spp infections. The terminologies of rare opportunistic yeast bloodstream infections (ROYBSIs) or micafungin breakthrough fungemia (MBF) or echinocandin non-susceptible yeast (ENSY) were used $(2,5,6)$.

\section{Corresponding Author: Mahir Kapmaz \\ E-mail: \\ mahirkapmaz@yahoo.com}

Received: December 09, 2019 Accepted: December 21, 2019 Published: December 25, 2019

Suggested citation: Kapmaz M, Tekin S, Doğan Ö, Keke Ş, Can F, Egönül O. Rare yeasts: emerging threat in immunocompromised patients. Infect Dis Clin Microbiol 2019; 3 : 165-172.

DOI: $10.36519 /$ idcm.2019.19030 
We present three cases of rare yeast fungemia and review the rare yeast infections with emphasis on diagnostic and therapeutic approach.

\section{Case 1. Saprochaete capitata fungemia}

A 73-year-old male with a history of myelodysplastic syndrome for nine years, who had a lower extremity amputation due to peripheral artery occlusion, and recurrent urosepsis with chronic renal failure was admitted to intensive care unit (ICU). After a successful resuscitation following cardiac arrest 20 days ago, he had been ventilated via tracheostomy and had tunnelled central venous catheher (CVC) for three months. While he had given micafungin for severe neutropenic fever with candiduria, S. capitata was isolated in his blood culture. His catheters were removed, and voriconazole was started. No fungus was recovered in his next cultures. After 14 days he died with septic shock due to an episode of P. aeruginosa bacteremia.

\section{Case 2. Saprochaete clavata fungemia}

A 21 years-old female with early recurrence of acute myeloid leukaemia after allogeneic stem cell transplantation, had several febrile neutropenia episodes. On the $30^{\text {th }}$ day of micafungin therapy, S. clavata was detected in her blood culture. Both amphotericin B and voriconazole were given, and central catheters were removed. No pathogen was detected in her blood cultures after ten days of treatment, but she died because of the intracranial haemorrhage three weeks later. To our knowledge, this was the first reported case of S. clavata fungemia in Turkey as a pathogen of MBF.

\section{Case 3. Kodamaea ohmeri fungemia}

A 43-year-old female, who was operated for colon tumour and had splenectomy in 2016, and received chemotherapy. Following removal of multiple nodules in the lung, the yeast-like fungus was reported in the blood culture. Micafungin was started, and CVC was removed. Pathology revealed nodular necrotizing granulomatous inflammation with oval or spherical, different sized fungi. K. ohmeri was clarified as the agent by VITEK2YST card (Biomerieux ${ }^{\circledR}$ ) and MALDI-TOF. She was treated for four weeks of intravenous fluconazole with cure.

\section{The incidence of Non-Candida yeasts}

The incidence of non-Candida and non-Cryptococcus yeasts in blood cultures was reported to be lower than candidemia. In a study, ROYBSIs were isolated from 94 blood culture specimens out of 41 patients, representing $0.18 \%$ of all positive blood cultures, $2.8 \%$ of all positive fungal blood cultures and 3.1\% (94/2984) of all positive blood culture for yeasts, so that the incidence of ROYBSIs was reported as 2.1 cases $/ 100,000$ patient-days (6). The incidence of ROYBSIs varied from $1.0 \%$ to $34 \%$ according to different countries, patient populations and medical settings. In a one-year surveillance study conducted in 25 hospitals across Asia, ENSY accounted for $6.7 \%$ of 2155 non-duplicate isolates from the blood (2).

The proportions of individual species (Cryptococcus, Trichosporon and Rhodotorula) vary according to the sample size of the studies or variations between countries. In reports from Belgium and Denmark, Saccharomyces cerevisiae was the predominant non-Candida isolate, while Trichosporon species in Asian countries, and Rhodotorula in a US single centre, Spain, and ARTEMiS study $(2,7)$. A retrospective review of ROYBSIs between 1998 and 2010 in a cancer centre in Texas, USA, revealed Rhodotorula in 21 (51\% of ROYBSIs) patients, Trichosporon in 8 (20\%) patients, S. cerevisiae in $8(20 \%)$ patients, Geotrichum in $2(5 \%)$ patients, Pichia anomala, and Malassezia furfur in one patient each (6).

The survey from a large (1900-bed) university hospital in Belgium reported incidence of fungemia and the distribution of species. They found that the annual incidence of fungemia ranged between

\section{HIGHLIGHTS}

- The yeasts other than Candida is emerging and clinicians should be alert about early distinction.

- MALDI-TOF might be considered as the most useful commercial system for identification

- A combination of L-AmB and voriconazole is the appropriate empirical therapy for especially in neutropenic patients. 
1.30 and 1.68 episodes per 10000 patient-days (on a total of 2680932 patient-days). S. cerevisiae was reported $0.7 \%$ (3/427 fungemia isolates) during this 2001-2005 period (8). Since only germ tube production and chromogenic media supplemented with API ID 32 were used with no available MALDI-TOF, no other yeast seems to be recognized in this survey.

Non-Candida fungemia was reported mostly as breakthrough infections, between 49\% (20/41 fungemia) and 59\% (10/17 cases) among fungemia studies $(6,9)$. This ratio was about $20 \%$ (52/258 cases) for Candida fungemia in cancer patients from European Organization for Research and Treatment of Cancer (EORTC) centres (9). Non-Candida fungemia had higher mortality than Candidemia (54.5\% versus $21.2 \%, P=0.03$ ) in a study of 110 episodes of fungemia with clinical sepsis from Kyoto, Japan, with the leading agents of C. neoformans and T. asahii (3).

\section{Risk factors}

Prior colonisation seems to be an important predictor of non-Candida fungemia. A study from Japan aimed to compare the non-Candida fungemia and candidemia in a tertiary hospital between 2004 and 2009, and the presence of collagen disease [odds ratio (OR) 9; 95\% confidence interval (CI) 1.58-51.4; $\mathrm{p}=0.01]$ or renal replacement therapy (OR 15; 95\% CI 3.06-73.4; P < 0.01) were found to be significantly more common in non-Candida fungemia group (3). While presence of solid malignancy was a risk factor for candidemia (0\% vs $45 \%, \mathrm{p}<0.01$ ), prior bacteremia was found to be a risk factor for non-candidemia attacks (63.6\% vs $32.3 \%, p=0.04)$. The presence of catheter was found in $51 \%$ of the patients with ROYBSIs, most of whom had solid or haematological malignancy (6).

\section{How to differentiate Candida from other yeasts in positive blood cultures?}

If Cryptococcus is suspected, urea test and inoculation in Niger culture medium should be applied (10-12). All Trichosporon isolates might be named in species level based on urease production and the presence of arthroconidia (10). Commercial systems, such as the Vitek Yeast Biochemical Card, API 20C, and API ID32C (bioMe'rieux ${ }^{\circledR}$ ) need an incubation period of 24 to $48 \mathrm{~h}$, and their application is mostly limited because of small databases (13).
The C. albicans germ tube antibody (CAGTA) test consists of an immunofluorescence assay to detect antibodies against an antigen of C. albicans, hwp1. Besides C. albicans, other Candida species are able to produce hwp1 to some degree. Whenever Candida hyphae invade body tissues, a positive serum CAGTA is supposed to be detected. Jimenez et al. reported that candidemia with a positive CAGTA should be considered as invasive candidiasis (14).

The combinations of CAGTA and (1-3)-b-D-glucan (BDG) or CAGTA and mannan antigens (Mn) had high NPV at the alternative cut-offs and could be useful in discontinuation of unnecessary empirical therapy in patients with suspected candidemia (15). The recommendations by European Conference on Infections in Leukemia (ECIL) for the biomarkers in diagnosis of invasive fungal diseases in leukemic patients and hematopoietic SCT recipients were at the moderate level for BDG detection in serum (BII), and the combined $\mathrm{Mn} / \mathrm{Anti}-\mathrm{Mn}$ testing in serum for hepatosplenic candidiasis (B III), and candidemia (C II). No recommendations were formulated for the use of PCR owing to a lack of standardization and clinical validation (16). According to the guideline of European Society of Clinical Microbiology and Infectious Diseases (ESCMID) the serum Mn/Anti-Mn was recommended for the diagnosis of candidemia but not for diagnosis of invasive candidiasis, and BDG was recommended to rule out Candida infection (12). On the other hand, findings suggest a possible role for BDG testing in diagnosis, and also treatment response of S. clavata infections (17).

With a protocol lasting 4 hours, rapid identification of Candida species from positive blood cultures by combining PCR and pyrosequencing of a small region of the $18 \mathrm{~S}$ rRNA gene is also proposed (18).

An oligonucleotide array system targeting the internal transcribed spacer (ITS) for rRNA genes was used to analyse 116 fungus-positive blood cultures from 105 patients, and high specificity and sensitivity were obtained compared to traditional methods (19). In a study by Hsiue et al., an array was developed to identify 77 species (16 genera) of medically relevant yeasts, including Cryptococcus, Rhodotorula, Saccharomyces, and Trichosporon species within 16-24 hours, and the sensitivity of $100 \%$ and the speci- 
ficity of $97 \%$ were obtained (20). MALDI-TOF was gradually implemented, and now is the backbone for the final identification. MALDI-TOF MS protocol was reported to be able to identify Fusarium, Exophiala, Saprochaete, and Trichosporon species directly from aliquots of positive BCs (20).

T2 magnetic resonance using nanoparticles has been approved for clinical usage (T2 Candida, T2 Biosystems, USA). The assay detects Candida species (C. albicans/C. tropicalis, C. parapsilosis, and C. krusei/C. glabrata) directly from positive blood samples, but not differentiate viable organisms. It also seems helpful for patients with candidemia under therapy. It has a sensitivity of $91 \%$ with a time to positivity of $4.4 \mathrm{hrs}$ and a limit of detection of $1-3 \mathrm{CFU} / \mathrm{mL}$ (21).

\section{Trichosporon sp.}

Trichosporon infection has a high mortality rate (53\%-76\%) and could be misdiagnosed as Candida infection (1). The cryptococcal latex test might result with false-positivity in patients with disseminated Trichosporon infection due to shared antigens (22). Trichosporon is the second most common yeast among the patients with fungemia in haematological patients and frequently the pathogen of breakthrough invasive fungal infection (bIFI) in patients receiving antifungal therapy. Trichosporon fungemia was significantly correlated with neutropenia than other ROYBSIs. Seven strains from 39 patients with haematological diseases with MBF between 20082015 were reported to be Trichosporon asahii (5).

In a review of 185 cases of Trichosporon fungemia, the number of cases increased from 1975 to 2014, especially over the past decade (1). The review revealed that a negative outcome was associated with a history of antimicrobial use, bacterial bloodstream co-infection, prophylactic/empirical antifungal therapy, Trichosporon beigelii infection, and receiving the antifungal regimen of amphotericin B/liposomal amphotericin B. A significantly greater proportion of patients with a positive outcome had fungemia without invasive tissue infection and received a voriconazole regimen or an amphotericin-triazole combined regimen. The significant positive outcome was also reported with patients who had recovered from neutropenia or after CVC removal (1).

\section{Saprochaete capitata (Bastoschzomyces capitatus)} Saprochaete spp. are filamentous fungi. In culture, dry, cottony colonies consisting of hyphae with arthroconidia were observed (23), which differs from the colonies of Candida spp. They were previously considered to be non-pathogenic. In microscope, the isolates produce hyphae, pseudohyphae, blastoconidia, arthroconidia and annelloconidia (24). Saprochaete spp. may colonize skin and mucosae and cause to infections in patients with underlying hematologic malignancies (25). In report from Spain, 20 out of 25 acute leukemia patients had fungemia with visceral dissemination with 30-day mortality of 52\% (26). In a report from Turkey, from 2012 to 2015 , a total of 20 S. capitata strains were isolated from onco-haematological and ICU patients in a university hospital (27). It was involved with many outbreaks and contaminated dairy products (25). Spondylodiscitis in a patient with solid organ tumour, fungemia in a non-neutropenic patient after aortic valve replacement, and opportunistic infections in polymyositis and dermatomyositis patients were also reported (28-30).

\section{Saprochaete clavata}

Saprochaete clavata (Geotrichum clavatum) is a rare, but still emerging yeast. It was thought to be frequently mistaken for G. capitatum and G. candidus on account of erroneous and limited databases of automated systems $(25,31)$. S. clavata was more rarely isolated from human samples than $\mathrm{S}$. capitatum. We summarized non-duplicate 56 cases of $S$. clavata infections reported in the literature (Table 1). A total of 37 of the patients had AML, and the fatality rate was $66 \%$ (Table 1 ).

In France, they represented $2.5 \%$ of the 8366 invasive yeast isolates in a prospective surveillance programme from 2002 to 2018, CNRMA. In this study, Saprochaete spp. was placed after Cryptococcus (14.5\%), and before Saccharomyces (0.7\%), Trichosporon spp. (0.6\%), Rhodotorula (0.5\%) (31).

S. clavata had been rarely reported in monocentric outbreaks in Europe until 2012. Then a multicentric outbreak in haematology wards in France, with 33 cases of S. clavata invasive infections was reported. Seventeen cases of them were diagnosed within two months period in three health care fa- 
Table 1. The cases infected with S.clavata, including our cases (references, 17, 23,32, 33, 39-47.

\begin{tabular}{|c|c|c|c|c|c|c|c|c|c|c|}
\hline Study & $\mathrm{n}$ & Sex (M/F) & $\begin{array}{l}\text { Cyta } \\
\text { Rabine } \\
\text { exposure }\end{array}$ & Diagnosis & Prophylaxis & $\begin{array}{l}\text { Culture } \\
\text { result }\end{array}$ & Infection site & $\begin{array}{l}\text { Empirical } \\
\text { therapy }\end{array}$ & $\begin{array}{l}\text { Directed } \\
\text { therapy }\end{array}$ & Outcome \\
\hline Vaux et al. & 30 & $15 / 15$ & $18 / 23$ & $\begin{array}{l}21 \mathrm{AML} \\
6 \mathrm{ALL}\end{array}$ & - & $\begin{array}{l}\text { Blood, } \\
\text { Respiratory }\end{array}$ & BSI, Pneumonia & $\begin{array}{l}\text { Caspo (16 } \\
\text { cases) }\end{array}$ & - & 24/30 died \\
\hline Picard et al. & 3 & $2 / 1$ & $3 / 3$ & $3 \mathrm{AML}$ & Posa & Blood & $\begin{array}{l}\text { Lung, intestine, } \\
\text { cardiac }\end{array}$ & Caspo & $\begin{array}{l}\mathrm{L} \text {-amb and } \\
\text { vori }\end{array}$ & $3 / 3$ died \\
\hline $\begin{array}{l}\text { Camus } \\
\text { et al. }\end{array}$ & 1 & $1 / 0$ & $1 / 1$ & $1 \mathrm{AML}$ & Posa & Blood & $\begin{array}{l}\text { Liver, } \\
\text { peritoneum }\end{array}$ & Caspo & Vori & Alive \\
\hline $\begin{array}{l}\text { Lacroix } \\
\text { et al }\end{array}$ & 2 & $2 / 0$ & $2 / 2$ & $2 \mathrm{AML}$ & No & $\begin{array}{l}\text { Blood, urine, } \\
\text { skin }\end{array}$ & & Ampho & $\begin{array}{l}\text { L-amb and } \\
\text { vori with } \\
\text { flusitozin }\end{array}$ & Alive \\
\hline Favre et al. & 1 & $1 / 0$ & & $\begin{array}{l}\text { Aplastic } \\
\text { anaemia }\end{array}$ & No & Blood, stool & $\begin{array}{l}\text { Liver, spleen, } \\
\text { lung, kidney, } \\
\text { bowel }\end{array}$ & Caspo & $\begin{array}{l}\text { L-Amb and } \\
\text { vori }\end{array}$ & Alive \\
\hline $\begin{array}{l}\text { Del Principe } \\
\text { et al. }\end{array}$ & 3 & $1 / 2$ & $3 / 3$ & $\begin{array}{l}2 \mathrm{AML} \\
1 \mathrm{NHL}\end{array}$ & Posa & Blood & $\begin{array}{l}\text { Lung, spleen, } \\
\text { liver }\end{array}$ & - & $\begin{array}{l}\text { L-Amb (high } \\
\text { dose) with } \\
\text { oral vori }\end{array}$ & Alive \\
\hline $\begin{array}{l}\text { Fernández } \\
\text { et al. }\end{array}$ & 1 & $1 / 0$ & & $\begin{array}{l}1 \\
\text { Lymphoma }\end{array}$ & - & Blood & Blood, skin & - & L-Amb & died \\
\hline $\begin{array}{l}\text { Buchta } \\
\text { et al. }\end{array}$ & 6 & $1 / 6$ & $6 / 6$ & $\begin{array}{l}5 \mathrm{AML}, 1 \\
\text { Lymphoma }\end{array}$ & - & $\begin{array}{l}\text { Blood } \\
\text { Stool, } \\
\text { wound, bile, } \\
\text { urine }\end{array}$ & $\begin{array}{l}\text { Blood } \\
\text { Disseminated, } \\
\text { Res }\end{array}$ & - & $\begin{array}{l}\text { Vori/ } \\
\text { LC-Amb/D- } \\
\text { Amb }\end{array}$ & $5 / 6$ died \\
\hline Liu et al. & 1 & $1 / 0$ & $0 / 1$ & ALL & Micafungin & $\begin{array}{l}\text { Blood, } \\
\text { hepatic }\end{array}$ & $\begin{array}{l}\text { Blood, } \\
\text { abdomen }\end{array}$ & Vori & L-Amb & Alive \\
\hline Leoni et al. & 1 & $1 / 0$ & $0 / 1$ & HSCT & $\begin{array}{l}\text { Low dosage } \\
\text { L-Amb }\end{array}$ & Blood & $\begin{array}{l}\text { Blood, skin, } \\
\text { pulmonary }\end{array}$ & Vori/L-Amb & Vori/L-Amb & Alive \\
\hline $\begin{array}{l}\text { Stanzani } \\
\text { et al. }\end{array}$ & 4 & $3 / 1$ & $1 / 4$ & $\begin{array}{l}2 \mathrm{AML}, 2 \\
\mathrm{NHL}\end{array}$ & $\begin{array}{l}\text { Micafungin, } \\
\text { Anidulafungin }\end{array}$ & $\begin{array}{l}\text { Blood, BAL, } \\
\text { Skin }\end{array}$ & $\begin{array}{l}\text { Blood, lung, } \\
\text { liver, spleen, } \\
\text { CNS }\end{array}$ & Vori/L-Amb & Vori/L-Amb & $1 / 4$ died \\
\hline Wee et al. & 1 & $0 / 1$ & $0 / 1$ & ALL & - & Blood & Blood, skin & Vori/L-Amb & Vori/ L-Amb & Alive \\
\hline $\begin{array}{l}\text { Pavone } \\
\text { et al. }\end{array}$ & 1 & $0 / 1$ & $0 / 1$ & $\begin{array}{l}\text { Kidney } \\
\text { transplant }\end{array}$ & Caspo & Blood & BSI & L-Amb & Vori/L-Amb & Died \\
\hline Our case & 1 & $0 / 1$ & $1 / 1$ & $\mathrm{AML}$ & Micafungin & Blood & BSI & L-Amb & Vori/L-Amb & Died \\
\hline
\end{tabular}

BSI: bloodstream infection, Caspo: caspofungin,
Vori: voriconazole,

L-Amb: liposomal amphotericin B,
D-Amb: Amphotericin- deoxycholate,

LC-Amb: lipid complex amphotericin 
cilities, suggesting a common source of contamination (32), but not proven. In this outbreak, S. clavata was recovered mostly from the blood (37/41, $90 \%)$. The case fatality rate on day 60 was reported as $66 \%$. The optimal therapy of invasive geotrichosis remains a challenge. Echinocandins are not recommended because of intrinsic resistance. Voriconazole, posaconazole (but not fluconazole), amphotericin B and 5-fluorocytosine are effective antifungal agents because of better in vitro and in vivo activities. Preventive antifungal posaconazole treatment, which seems to be effective in reducing the number of proven invasive fungal infections in haematological patients, did not prevent the development of this severe infection in some cases (23). This could be explained by subtherapeutic dosage due to gastrointestinal elimination of posaconazole suspension (23). A combination of amphotericin B with voriconazole could be better than voriconazole alone, but still remains undetermined (33). Highdose liposomal amphotericin B might be more effective than the standard dose.

\section{Kodamea ohmeri}

Kodamaea ohmeri is from Saccharomycetaceae family, and was formerly known as Pichia ohmeri. It is an opportunistic pathogen among infants and older age groups with immunosuppression, hematologic malignancy or chronic lung disease with CVC source (34-36). K. ohmeri can present with different clinical features such as peritonitis, endocarditis, mediastinal mass, sepsis, oropharyngeal, and urinary tract infections $(35,37)$. Kodamaea ohmeri has a fermentation capability, therefore used in the preparation of pickles (35). K. ohmeri had low MIC levels to all antifungal agents except fluconazole in few cases. K. ohmeri and C. tropicalis are similar phenotypically. In a pediatric centre, thirty-eight out of 148 (25.7\%) phenotypically identified C. tropicalis isolates were confirmed as $\mathrm{K}$. ohmeri by using advanced techniques. K. ohmeri has the potential to cause hospital outbreaks. Piperacillin-tazobactam use was reported to be significantly associated with the K. ohmeri fungaemia (38). Unlike the pathogens of ROYBSIs, K. ohmeri was reported to have low MICs to echinocandins. In our case 3, the K.ohmeri was susceptible to fluconazole, and the patient was cured with fluconazole.

\section{CONCLUSION}

Identification of the yeasts with conventional methods might be late, which may result with the inappropriate use of echinocandins in patients with ENSY fungemia. ROYBSIs should be taken into consideration when yeast growth in blood culture of a patient previously treated with an antifungal, especially an echinocandin. The slow growth of yeast in solid media, their colony type, and urease positivity might be helpful in the differentiation. Apart from molecular methods, MALDI-TOF might be considered as the most useful commercial system for identification of ROYBSIs. A combination of $\mathrm{L}$-AmB and voriconazole might be the appropriate empirical therapy for MBF, especially in neutropenic patients. L-AmB might be reasonable for MBF in non-neutropenic patients.
Peer-review: Externally peer-reviewed

Author Contributions: Concept-M.K., Ö.E., F.C.; Design - M.K., Ö.E.; Supervision - F.C., Ö.E.; Materials - M.K., S..K., S.T.; Data Collection and/or Processing - M.K., S..K., S.T.; Analysis and/or Interpretation - M.K., Ş.K.; Literature Review - M.K., S.T., Ö.D.; Writer - M.K., Ş.K., Ö.E.; Critical Reviews - Ö.E., F.C., Ö.D.
Conflict of Interest: The authors have no conflict of interest to declare.

Financial Disclosure: The authors declared that this study has received no financial support. 


\section{REFERENCES}

1 Liao Y, Lu X, Yang S, Luo Y, Chen Q, Yang R. Epidemiology and outcome of Trichosporon fungemia: a review of 185 reported cases from 1975 to 2014. Open Forum Infect Dis 2015; 2: ofv141.

2 Lin SY, Lu PL, Tan BH, Chakrabarti A, Wu UI, Yang JH, et al. Asia Fungal Working Group (AFWG). The epidemiology of non-Candida yeast isolated from blood: The Asia Surveillance Study. Mycoses 2019; 62: 112-120.

3 Yamamoto M, Takakura S, Hotta G, Matsumura Y, Matsushima A, Nagao M, et al. Clinical characteristics and risk factors of non-Candida fungaemia. BMC Infect Dis 2013; 13: 247.

4 Paiva JA, Pereira JM, Tabah A, Mikstacki A, de Carvalho FB, Koulenti D, et al. Characteristics and risk factors for 28-day mortality of hospital acquired fungemias in ICUs: data from the EUROBACT study. Crit Care 2016; 20: 53.

5 Kimura M, Araoka H, Yamamoto H, Nakamura S, Nagi M, Yamagoe S, et al. Micafungin breakthrough fungemia in patients with hematological disorders. Antimicrob Agents Chemother 2018; 62: e02183-17

6 Chitasombat MN, Kofteridis DP, Jiang Y, Tarrand J, Lewis RE, Kontoyiannis DP. Rare opportunistic (non-Candida, non-Cryptococcus) yeast bloodstream infections in patients with cancer. J Infect 2011; 64: 68-75.

7 Marcos-Zambrano LJ, Escribano P, Sánchez C, Muñoz P, Bouza E, Guinea J. Antifungal resistance to fluconazole and echinocandins is not emerging in yeast isolates causing fungemia in a Spanish tertiary care center. Antimicrob Agents Chemother 2014; 58: 4565-72.

8 Lagrou K, Verhaegen J, Peetermans WE, De Rijdt T, Maertens J, Van Wijngaerden E. Fungemia at a tertiary care hospital: incidence, therapy, and distribution and antifungal susceptibility of causative species. Eur J Clin Microbiol Infect Dis 2007; 26: 541-7.

9 Cornely OA, Gachot B, Akan H, Bassetti M, Uzun O, Kibbler C, et al. Epidemiology and outcome of fungemia in a cancer Cohort of the Infectious Diseases Group (IDG) of the European Organization for Research and Treatment of Cancer (EORTC 65031) Clin Infect Dis 2015; 61 (3): 324-31.

10 A. M. Montoya, G. M. González Trichosporon spp: an emerging fungal pathogen. Medicina Universitaria 2014; 16(62): 37-43.

11 Chakrabarti A, Chatterjee SS, Rao KL, Zameer MM, Shivaprakash MR, Singhi S, et al. Recent experience with fungaemia: change in species distribution and azole resistance, Scand J Infect Dis 2009; 41:275-84.

12 Cuenca-Estrella M, Verweij PE, Arendrup MC, Arikan-Akdagli S, Bille J, Donnelly JP, et al. ESCMID guideline for the diagnosis and management of Candida diseases 2012: diagnostic procedures. Clin Microbiol Infect. 2012; 18 Suppl 7:9-18

13 Leaw SN, Chang HC, Barton R, Bouchara JP, Chang TC. Identification of medically important Candida and non-Candida yeast species by an oligonucleotide array. J Clin Microbiol 2007; 45:2220-9.

14 Martínez-Jiménez MC, Muñoz P, Guinea J, Valerio M, Alonso R, Escribano P, et al. Potential role of Candida albicans germ tube antibody in the diagnosis of deep-seated candidemia. Med Mycol 2014; 52: 270-5

15 Martínez-Jiménez MC, Muñoz P, Valerio M, Alonso R, Martos C, Guinea J, et al. Candida biomarkers in patients with candidaemia and bacteraemia. J Antimicrob Chemother 2015; 70 : 2354-61.

16 Marchetti O, Lamoth F, Mikulska M, Viscoli C, Verweij P, Bretagne S, et al. ECIL recommendations for the use of biological markers for the diagnosis of invasive fungal diseases in leukemic patients and hematopoietic SCT recipients. Bone Marrow Transplant 2012; 47: 846-54.

17 Del Principe MI, Sarmati L, Cefalo M, Fontana C, De Santis G, Buccisano F, et al. A cluster of Geotrichum clavatum (Saprochaete clavata) infection in haematological patients: a first Italian report and review of literature. Mycoses 2016; 59 (9): 594-601.

18 Quiles-Melero I, García-Rodriguez J, Romero-Gómez MP, Gómez-Sánchez P, Mingorance J. Rapid identification of yeasts from positive blood culture bottles by pyrosequencing. Eur J Clin Microbiol Infect Dis 2011; 30: 21-4.

19 Hsiue HC, Huang YT, Kuo YL, Liao CH, Chang TC, Hsueh PR. Rapid identification of fungal pathogens in positive blood cultures using oligonucleotide array hybridization. Clin Microbiol Infect 2010;16(5): 493-500.

20 de Almeida JN Júnior, Sztajnbok J, da Silva AR Junior, Vieira VA, Galastri AL, Bissoli L, et al. Rapid identification of moulds and arthroconidial yeasts from positive blood cultures by MALDI-TOF mass spectrometry. Med Mycol 2016; 54: 885-9.

21 Patterson TF, Donnelly JP. New concepts in diagnostics for invasive mycoses: non-culture-based methodologies. J Fungi (Basel) 2019; 5: 9.

22 Ebright JR, Fairfax MR, Vazquez JA. Trichosporon asahii, a non-Candida yeast that caused fatal septic shock in a patient without cancer or neutropenia. Clin Infect Dis 2001; 33: E28-30.

23 Camus V, Thibault ML, David M, Gargala G, Compagnon P, Lamoureux F, et al. Invasive Geotrichum clavatum fungal infection in an acute myeloid leukaemia patient: a case report and review. Mycopathologia 2014; 177(5-6): 319-24.

24 Arendrup MC, Boekhout T, Akova M, Meis JF, Cornely OA, Lortholary O; European Society of Clinical Microbiology and Infectious Diseases Fungal Infection Study Group; European Confederation of Medical Mycology. ESCMID and ECMM joint clinical guidelines for the diagnosis and management of rare invasive yeast infections. Clin Microbiol Infect 2014; 20 Suppl 3:76-98.

25 Desnos-Ollivier M, Blanc C, Garcia-Hermoso D, Hoinard D, Alanio A, Dromer F. Misidentification of Saprochaete clavata as Magnusiomyces capitatus in clinical isolates: utility of internal transcribed spacer sequencing and matrix-assisted laser desorption ionization-time of flight mass spectrometry and importance of reliable databases. J Clin Microbiol 2014; 52(6):2196-8.

26 Martino R, Salavert M, Parody R, Tomás JF, de la Cámara R, 
Vázquez L, et al. Blastoschizomyces capitatus infection in patients with leukemia: report of 26 cases. Clin Infect Dis 2004; 38(3): 335-41.

27 Koç AN, Atalay MA, Timur D, Demir G, Kaynar L. Molecular epidemiology and antifungal susceptibility of Saprochaete capitata (Blastoschizomyces capitatus) isolates causing nosocomial infection in Kayseri/Turkey. Infect Dis (Lond) 2016; 48(8): 596-603.

28 Cavanna C, Lallitto F, Mangione F, Tamarozzi F, Marone P, Ceriana P. Fungemia due to Saprochaete capitata in a non-neutropenic patient hospitalized in an intensive care unit after cardiac surgery. J Mycol Med 2017; 27(2):281-284.

29 Celik AD, Ozaras R, Kantarcioglu S, Mert A, Tabak F, Ozturk R. Spondylodiscitis due to an emergent fungal pathogen: Blastoschizomyces capitatus, a case report and review of the literature. Rheumatol Int 2009; 29(10):1237-41.

30 Marie I, Ménard JF, Hachulla E, Chérin P, Benveniste O, Tiev K, et al. Infectious complications in polymyositis and dermatomyositis: a series of 279 patients. Semin Arthritis Rheum 2011; 41(1):48-60.

31 CNRMA 2018 activity report. https://www.pasteur.fr/fr/ sante-publique/CNR/les-cnr/mycoses-invasives-antifongiques/rapports-d-activite ( 25/11/2019)

32 Vaux S, Criscuolo A, Desnos-Ollivier M, Diancourt L, Tarnaud C, Vandenbogaert M, et al. Geotrichum Investigation Group. Multicenter outbreak of infections by Saprochaete clavata, an unrecognized opportunistic fungal pathogen. MBio 2014; 5(6). pii: e02309-14.

33 Favre S, Rougeron A, Levoir L, Pérard B, Milpied N, Accoceberry I, et al. S. Saprochaete clavata invasive infection in a patient with severe aplastic anemia: Efficacy of voriconazole and liposomal amphotericin B with adjuvant granulocyte transfusions before neutrophil recovery following allogeneic bone marrow transplantation. Med Mycol Case Rep 2016;11:21-3.

34 Fernández-Ruiz M, Guinea J, Puig-Asensio M, Zaragoza Ó, Almirante B, Cuenca-Estrella M, et al. CANDIPOP Project; GEIH-GEMICOMED (SEIMC) and REIPI. Fungemia due to rare opportunistic yeasts: data from a population-based surveillance in Spain. Med Mycol 2017; 55(2):125-136.

35 Santino I, Bono S, Borruso L, Bove M, Cialdi E, Martinelli D, et al. Kodamaea ohmeri isolated from two immunocompromised patients: first report in Italy. Mycoses 2013; 56:179-81.

36 Alvarado Socarras J, Rojas Torres JP, Vargas Soler JA, Guerrero C. Kodamaea ohmeri infection in a newborn with a mediastinal mass. Arch Argent Pediatr 2016 Oct 1; 114(5):e319-22.

37 Tashiro A, Nei T, Sugimoto R, Watanabe A, Hagiwara J, Takigu- chi $\mathrm{T}$, et al. Kodamaea ohmeri fungemia in severe burn: Case study and literature review. Med Mycol Case Rep 2018; 22: 21-23.

38 Chakrabarti A, Rudramurthy SM, Kale P, Hariprasath P, Dhaliwal M, Singhi S. Epidemiological study of a large cluster of fungaemia cases due to Kodamaea ohmeri in an Indian tertiary care centre. Clin Microbiol Infect 2014; 20(2):083-9.

39 Lacroix C, Brethon B, Boissel N, Desnos M, Leblanc T, Raffoux, E, et al. Geotrichum clavatum an emerging pathogen responsible for invasive infection in two neutropenic leukemia patients. abstract No: P180 Full conference title: 3rd Trends in Medical Mycology 2007, Italy, NULL Conference abstracts

40 Picard M, Cassaing S, Letocart P, Verdeil X, Protin C, Chauvin P, et al. Concomitant cases of disseminated Geotrichum clavatum infections in patients with acute myeloid leukemia. Leuk Lymphoma 2014; 55(5):1186-8.

41 Salgüero Fernández I, Nájera Botello L, Orden Martinez B, Roustan Gullón G. Disseminated fungemia by Saprochaete clavata. Enferm Infecc Microbiol Clin 2018 13. pii: S0213005X(18)30190-3.

42 Buchta V, Bolehovská R, Hovorková E, Cornely OA, Seidel D, Žák P Saprochaete clavata Invasive Infections - A New Threat to Hematological-Oncological Patients. Front Microbiol 2019; 10:2196

43 Liu X, Zhou W, Jiang Y, Kuang L. Invasive Fungal Infection Caused by Geotrichum clavatum in a Child with Acute Leukemia: First Documented Case from Mainland China. Jpn J Infect Dis 2019; 72(2):130-132.

44 Leoni M, Riccardi N, Rotulo GA, Godano E, Faraci M, Bandettini $\mathrm{R}$, et al. Magnusiomyces clavatus infection in a child after allogeneic hematotopoetic stem cell transplantation: Diagnostic and therapeutic implications. Med Mycol Case Rep 2018; 23:65-67.

45 Stanzani M, Cricca M, Sassi C, Sutto E, De Cicco G, Bonifazi $\mathrm{F}$, et al. Saprochaete clavata infections in patients undergoing treatment for haematological malignancies: A report of a monocentric outbreak and review of the literature. Mycoses 2019; 62(12):1100-1107.

46 Wee LWY, Ling HY, Chong CY, Soe MN, Koh MJA. A rare case of purpuric rash caused by Saprochaete clavata in a pediatric patient with acute leukemia. Pediatr Dermatol 2019; 36(6):990991.

47 P. Pavone, A. Oliva, G. Raponi, F. Pugliese, S. Martelli, P. Celli, et al. Disseminated fungal infection due to Saprochaete clavata in a kidney transplant recipient. Journal de Mycologie Médicale 2019; 29, 278-82. 